\title{
Aircraft Path Planning under Adverse Weather Conditions
}

\author{
Xie Z. and Zhong Z. W. \\ School of Mechanical and Aerospace Engineering, Nanyang Technological University, Republic of Singapore
}

\begin{abstract}
In recent years, flight safety is still one of the main issues for all airlines. En route civil airplanes may encounter adverse weather conditions. Some fatal airplane accidents happened because of the weather disturbance. Moreover, we should also design path to avoid the prohibited area. Therefore a good path planning algorithm plays an increasingly important role in air traffic management. An efficient path planning algorithm can help the plane to avoid severe weather conditions, restricted areas and moving obstacles to ensure the safety of the cabin crews and passengers. Here, we build our algorithm based on the A* search algorithm. Moreover, our algorithm can also find the path with least energy costs. As a result, our algorithm can improve the safety operation of the airplanes and reduce the workload of pilots and air traffic controllers.
\end{abstract}

\section{Introduction}

In recently years, some airplane accidents happened around the world. How to ensure the air traffic safety operation has been a main topic in Air Traffic Management (ATM). Researchers around the globe try their best to think of ways to not only increase the safety but also improve the efficiency of the airplane. As we can see in Fig. 1, adverse weather is one of the main factors which can cause the fatal aircraft incidents. How to avoid the adverse weather and create a new efficient path is our research goal. Adverse weather will also have effect on the visibility of the aircraft. [1] We also take into consideration of the effect from other aircraft in the same airspace and limitations of airspace restriction areas. The prohibited areas can also cause danger to the civil airplanes. For instance, civil airplanes can interrupt military drills or can be hit by missiles and fighters if they enter military prohibited areas or war zones.

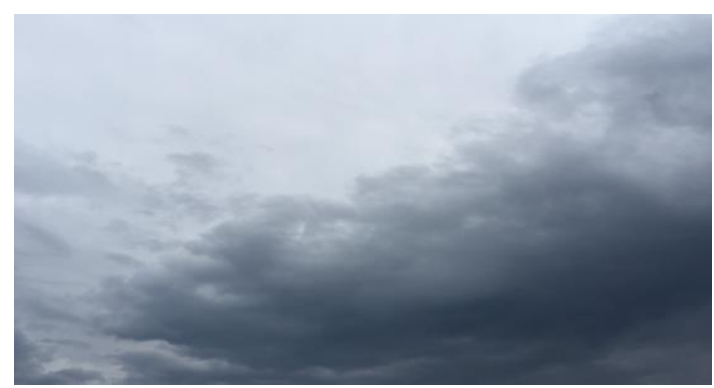

Figure 1. Adverse Weather condition

Free flight concepts [2] was first introduced by Radio Technical Commission for Aeronautics (RCTA) in 1995. As shown in Fig. 2, free flight means the en route aircraft pilots are more responsible for the separation of aircraft while reducing the workload of air traffic controllers
(ATCs), but in case of emergency, ATCs should intervene if pilots fail to execute separations. This concepts can free the ATCs so that they can focus more on the users' request.

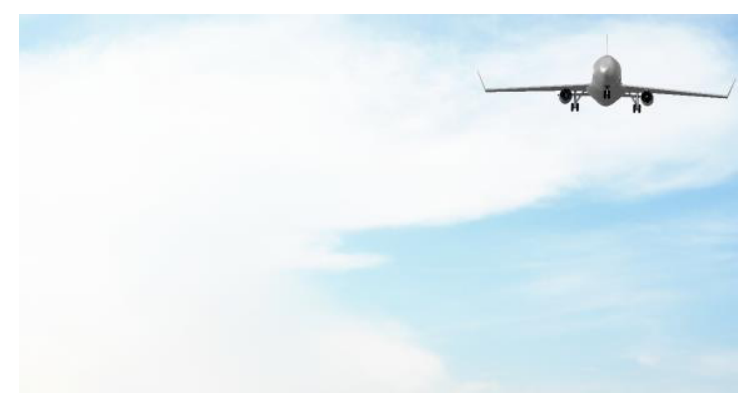

Figure 2. Pilots should have more freedom and responsibility to separate and maneuver

Christina [3] introduced a novel 4D trajectory optimization method that creates a path based on adverse weather data. This application can successfully reduce the detour and fuel cost. They created a reference route for comparison of the detour deduction. But the author only considered storm cell related circumnavigation, other weather and traffic conflicts. Airspace restrictions were still not taken into consideration yet.

Wang [4] developed an air route network node optimization method in order to avoid the tree areas, including prohibited area, restricted area and danger area. A cellular automata model was deployed to find the shortest path for optimization problems. The authors used a case study to test the effectiveness of the model. In the end, the methods can reduce the conflict risks of cross network and decrease flight costs and the nonlinear coefficient.

The FAA rules [5] state that the minimum separation of en route flights is $5 \mathrm{nmi} ; 3 \mathrm{nmi}$ for lower level terminal 
space. When aircraft are detected in the same airspace, the minimum separation must be satisfied in case of emergency. In our simulation case study, we use single dot to represent aircraft which share the same airspace. We take the minimum separation into consideration when optimizing the route under specific conditions.

A* search algorithm was first introduced by Hart and Nilsson [6] in 1968. It can transfer heuristic information to mathematical theory of graph searching. Also A* search algorithm is proved to be effective in finding the optimal route while examining the minimum number of nodes. The cost function contains two part, one is the cost from the initial position to current position; another one is the estimate cost from the preferred goal to the current position. We build our algorithm based on $A^{*}$ search algorithm and use our own cost function to get the most efficient solution.

Soltani [7] compared Dijkstra, A* and Genetic Algorithm (GA) for path planning in constructive sites. The objective function is combined of shortest path, high visibility and lowest risks. The three path planning methods are compared in time consumption, accuracy and complexity. He found that $\mathrm{A}^{*}$ search algorithm will be admissible if the distance from the current position to the preferred goal is always available by heuristic function $\mathrm{h}$. This is the restriction of $\mathrm{A}^{*}$ search algorithm that can cause to overestimate the cost to the goal.

Garau [8] utilized $A^{*}$ search algorithm on path planning of unmanned underwater vehicles in a real Mediterranean Sea situation. The aim of their research is to find the optimal energy saving path based on a current field model and satellite altimetry. Moreover, the vehicle is capable of obstacle avoidance. The results show that the path planner is robust and efficient for the autonomous underwater vehicle to replan a path to the destination with shorter time and less energy cost.

Adem [9] compared $A^{*}$ search algorithm with GA in dynamic mobile robot path planning. He found that GA performed better than conventional $A^{*}$ search algorithm both in travelling distance and computation time. Also, modified $\mathrm{A}^{*}$ search algorithm has better computation time but it is easy to reach a local minimum. GA can find the global optimal solution efficiently.

Chee and Zhong [10] used a nonlinear complementary filter to estimate the attitude of unmanned aerial vehicle (UAV) and implemented a reactive quad-directional algorithm to avoid obstacles. The results show the UAV can achieve attitude control and navigate open environment autonomously with obstacle avoidance capability.

Xie and Zhong [11] developed methods for the unmanned surface vehicle (USV) to create path automatically to avoid the obstacles on the sea surface. The simulation results in MATLAB show that the USV can find the most efficient path and lead the vehicle to the destination.

\section{Methodology}

As we can see in Fig. 3, a commercial airplane encounters an adverse weather condition. It may threaten the on-board equipment and also it may lead to the operation failure of the pilots. This is a dangerous situation which may cause a fatal disaster. We build our own algorithm based on the civil flight circumstance and simulate the situation using MATLAB when an airplane is under an adverse weather condition and airspace limitations. Paul [12] of Mathworks also created a MATLAB tutorial to compute the shortest path to the goal location using the $A^{*}$ search algorithm to help users better understand the function of the $\mathrm{A}^{*}$ search algorithm.

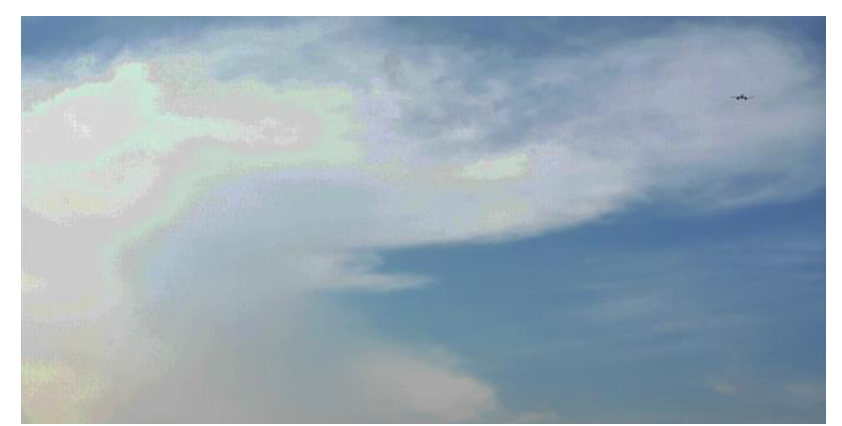

Figure 3. Civil airplane under an adverse weather condition

Air flight path planning is different from ground vehicle path planning. We trim the $\mathrm{A}^{*}$ algorithm to make it more fit to the en route environment. We change the optimization function to consider the deviation and turning angle as well.

\subsection{Rules}

Flight routes must also obey the following rules for the safety of aircraft and restriction of the airspace.

- Restricted or prohibited area cannot be violated.

- Weather conditions are forecasted and updated.

- The minimum distance between two aircraft en route should be 5 nmi [5].

- Adverse weather should be avoided for safety reasons [3].

- Find the least cost to reach the minimum fuel burning goal.

- The airplane has the ability of obstacles avoidance.

\subsection{Algorithm}

We use a grid to describe a $60 \times 60 \mathrm{nmi}$ area. One unit means $2 \mathrm{nmi}$. The plane starts from the start gate and ends at the end gate. The start gate and end gate can be chosen randomly before the simulation. The obstacles including the restricted areas, adverse weather areas and other aircraft are randomly distributed as well. The function of the algorithm is to avoid all the obstacles and satisfy the rules at the same time. The optimal path will be shown in black line and the obstacles are shown as red blocks.

The basic $A^{*}$ search algorithm objective function is as follows [6].

$$
f(n)=h(n)+g(n)
$$

$f(n)$ stands for the optimization function. 
$h(n)$ stands for the estimate cost from the current position to the final position.

$g(n)$ stands for the cost for reaching the current position from the initial position.

\subsubsection{Optimization function}

Based on the flight route requirement, we build our own optimization function. The function can be seen below in Eq. 2.

$$
F(n)=H(n)+G(n)+A(n)+D(n)
$$

$F(n)$ stands for the objective function.

$H(n)$ stands for the estimate cost from current position to the final position.

$G(n)$ stands for the cost for reaching the current position from the start position.

$A(n)$ is the turning angle of the aircraft.

$D(n)$ is the route deviation from the reference route.

Our goal is to get the smallest value of $F(n)$, but at the same time, the optimised route must also follow the restrictions and limitations to ensure the safe operation of the flight route.

\subsubsection{Restrictions and limitations}

As shown in Eq. 3, the turning angle for the airplane should not exceed the maximum turning angle [13].

$$
A(n)<A_{\max }
$$

As shown in Eq. 4, the route deviation should also be within a limit. It means the airplane should not deviate from its original path too much.

$$
D(n)<D_{\max }
$$

As can be seen in Eq. 5, the distance between two aircraft which share the same airspace should be at least 5 nmi [5].

$$
\operatorname{Dist}(x, y)>5 n m i
$$

As can be seen in Eq. 6, the cost function should achieve the least value among all the other entries.

$$
F\left(n^{*}\right)<F(n), n \in N
$$

\section{Results analysis and discussion}

\subsection{Case 1}

As can be seen in Fig. 4, in case 1, the aircraft starts from the initial position which is located at $(0,22.5)$ of the grid map and ends at the final position which is located in the lower right corner of the map. The obstacles are marked with pink circles in our simulation. The squares with the pink circles are closed for aircraft to pass. In this situation, the closed squares include prohibited area, adverse weather and moving aircraft. The black line shows the optimal path with the least energy cost and travelling distance which is generated by the algorithm. We can see that the algorithm finds the optimal path while avoiding the adverse weather, prohibited areas and other aircraft. The two aircraft which share the same airspace also are separated further than the minimum safety distance, which is $5 \mathrm{nmi}$. This autonomously generated path to avoid the moving aircraft can reduce the workloads of the ATCs and ensure the safe operation of airspace.

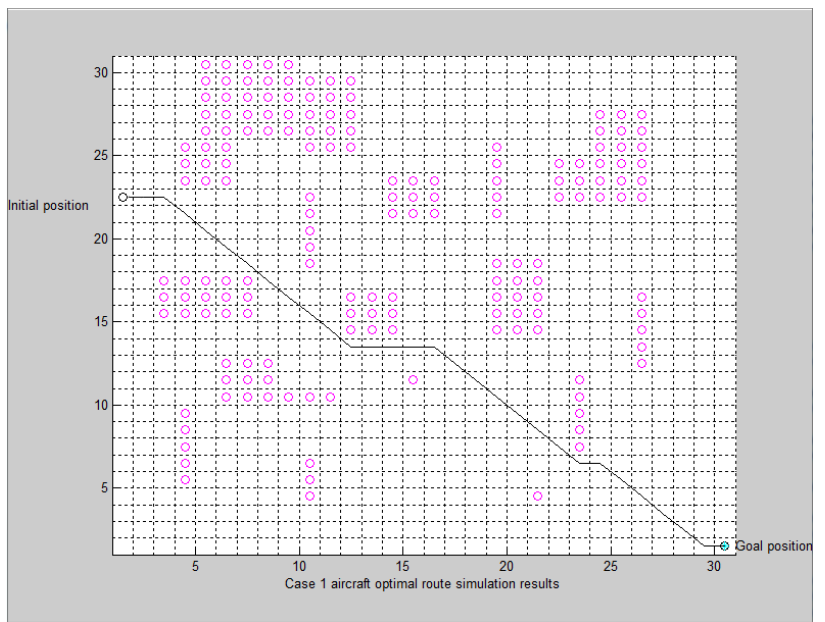

Figure 4. Simulation results of case 1

\subsection{Case 2}

Scenario 2 is designed for the unmanned vehicle (UV). The UV starts from the top left corner and ends at the lower right corner. This time the obstacles only include adverse weather and prohibited areas. The black line shows the optimal path in this case. We can clearly see in Fig. 5 that the path is the shortest and has the least deviation from the original path. Finally, the UV reaches the destination accurately.

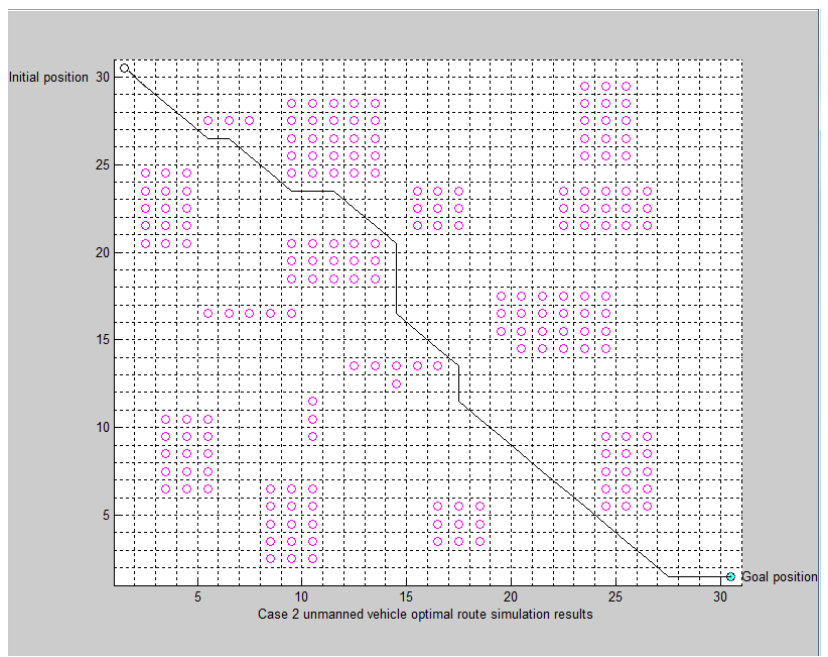

Figure 5. Case 2 simulation results

\section{Conclusion}

Our path planning algorithm based on the $A^{*}$ search algorithm can successfully find the most efficient path while avoiding the hazardous weather and airspace 
conditions. Also the obstacles can be randomly distributed so that we can have as many scenarios as possible to test the efficiency of our algorithm. The algorithm can find the optimal path with the least travelling distance and least energy cost while avoiding prohibited areas, adverse weather and surrounding aircraft. In conclusion, it can benefit ATM in decreasing the chance of accidents caused by bad weather and other conflicts, saving energy consumption and making the route more eco-friendly.

\section{Acknowledgement}

This research was sponsored by the ATMRI of NTU and CAAS via ATMRI Project No. 2014-D2-ZHONG for Regional Airspace Capacity Enhancement - ASEAN Pilot.

\section{References}

1. Xie, Z. and Z.W. Zhong, Simulated Civil Airplane Visual Conspicuity Experiments During Approaching and Departure in the Airport Vicinity, in 7th International Conference on Intelligent Systems, Modelling and Simulation. Bangkok, (2016).

2. Endsley, M.R., et al., Effect of free flight conditions on controller performance, workload, and situation awareness, DTIC Document, (1997).

3. Schilke, C. and P. Hecker. Dynamic Route Optimization Based on Adverse Weather Data. in Fourth SESAR Innovation Days.

4. Wang, S.-j. and Y.-h. Gong, Research on air route network nodes optimization with avoiding the three areas. Safety Science. 66: 9-18, (2014).
5. 7110.65U, F.O., Para 5-5-4 "[Radar Separation] Minima. FAA, (2013).

6. Hart, P.E., N.J. Nilsson, and B. Raphael, A Formal Basis for the Heuristic Determination of Minimum Cost Paths. Systems Science and Cybernetics, IEEE Transactions on. 4(2): 100-107, (1968).

7. Soltani, A.R., et al., Path planning in construction sites: performance evaluation of the Dijkstra, $A$ *, and $G A$ search algorithms. Advanced Engineering Informatics, 16(4): 291-303, (2002).

8. Garau, B., et al., Path planning for autonomous underwater vehicles in realistic oceanic current fields: Application to gliders in the western mediterranean sea. Journal of Maritime Research, 6(2): 5-22, (2014).

9. Tuncer, A. and M. Yildirim, Dynamic path planning of mobile robots with improved genetic algorithm. Computers \& Electrical Engineering,. 38(6): 15641572, (2012).

10. Chee, K.Y. and Z.W. Zhong, Control, navigation and collision avoidance for an unmanned aerial vehicle. Sensors and Actuators A: Physical. 190: 6676, (2013).

11. Xie, Z. and Z.W. Zhong, Unmanned Vehicle Path Optimization Based on Markov Chain Monte Carlo Methods. Applied Mechanics \& Materials, 829, (2016).

12. Premakumar, P. A* (A Star) search for path planning tutorial. 2010; Available from: http://www.mathworks.com/matlabcentral/fileexchan ge/26248-a---a-star--search-for-path-planningtutorial.

13. Phillips, W.F., Mechanics of flight. 2004: John Wiley \& Sons. 